\title{
MicroRNA-93 mediates cabergoline resistance by targeting ATG7 in prolactinoma
}

\author{
Zerui Wu ${ }^{1, *}$, Lin Cai ${ }^{1, *}$, Jianglong Lu1,*, Chengde Wang ${ }^{1}$, Jiaqing Guan ${ }^{1}$, Xianbin Chen ${ }^{1}$, Jinsen Wu ${ }^{1}$, Weiming Zheng ${ }^{1}$, \\ Zhebao Wu2 ${ }^{2}$ Qun Li1 and Zhipeng Su${ }^{1}$
}

1Department of Neurosurgery, First Affiliated Hospital of Wenzhou Medical University, Wenzhou, China

2Department of Neurosurgery, Ruijin Hospital, Shanghai Jiao Tong University School of Medicine, Shanghai, China

Correspondence should be addressed to Q Li or Z Su: garylina@126.com or drsuzhipeng@163.com

*(Z Wu, L Cai and J Lu contributed equally to this work)

\begin{abstract}
To date, the management of dopamine agonist (DA)-resistant prolactinomas remains a major clinical problem. Previously, we determined that miRNA-93 expression increases in DA-resistant prolactinomas; however, the role of miRNA-93 in the DA resistance remains largely unexplored. Hence, this study aimed to investigate the susceptibility of tumor cells to cabergoline (CAB) and the autophagy changes in MMQ and GH3 cells after miRNA-93 overexpression or inhibition. We used bioinformatics to identify the potential target of miRNA-93. Subsequently, we analyzed the correlation between miRNA-93 and autophagy-related 7 (ATG7) using protein expression analysis and luciferase assays. Furthermore, the change in the effect of miRNA-93 was measured after ATG7 overexpression. miRNA-93 expression was elevated in DA-resistant prolactinomas, whereas the expression of its identified target, ATG7, was downregulated. miRNA-93 overexpression suppressed the cytotoxic effect of CAB in MMQ and GH3 cells. In contrast, miRNA-93 downregulation enhanced CAB efficiency and promoted cell autophagy, eventually resulting in apoptosis. These results were further confirmed in in vivo xenograft models in nude mice. ATG7 overexpression could reverse the inhibitory effect of miRNA-93 on CAB treatment. Taken together, our results suggest that miRNA-93 mediates CAB resistance via autophagy downregulation by targeting ATG7 and serves as a promising therapeutic target for prolactinoma.
\end{abstract}
Key Words
- miRNA-93
- prolactinoma
- ATG7
- drug resistance
- cabergoline
- autophagy

\section{Introduction}

Prolactinomas are the most common subtype of pituitary adenomas (proportion, 40-66\%), with a prevalence of 6-10 cases per 100,000 per year (Gillam et al. 2006, Wu et al. 2006). Dopamine agonists (DAs), such as bromocriptine (BRC) and cabergoline (CAB), are the leading treatment options for prolactinomas. $\mathrm{CAB}$ is more effective, has fewer side effects and is longer lasting than BRC in shrinking the tumor volume and suppressing prolactin (PRL) hypersecretion (Colao et al. 2007, Molitch 2010, Wu et al. 2016b). However, approximately $11 \%$ of patients with prolactinomas are resistant to $\mathrm{CAB}$ (Molitch 2003). Thus, the treatment of drug-resistant prolactinomas remains a challenging clinical problem.

miRNAs are a family of endogenous, small noncoding, single-stranded RNA, with approximately 16-29 nucleotides in length (Adams et al. 2017) and regulate the target gene expression by binding to the 3 '-untranslated 
region (UTR) of the mRNA (Zeng et al. 2017). There are many reports of miRNA-mediated drug resistance in cancers (Fanini \& Fabbri 2016), including breast cancer (Zhang et al. 2015), osteosarcoma (Duan et al. 2017), pancreatic cancer (Neesse \& Gress 2015) and myeloma (Ahmad et al. 2014). Recently, some studies have reported that miRNA-93 regulates chemotherapy resistance in colorectal carcinoma, breast cancer and ovarian cancer (Srivastava et al. 2015, Asaduzzaman et al. 2017, Wang et al. 2017). Previously, we reported that miRNA-93 expression increases in DA-resistant prolactinomas (Wu et al. 2014); however, the role of miRNA-93 in drug resistance remains largely unclear.

Autophagy, a conserved protein and cell cargo degradation pathway in eukaryotes, is essential for cell survival, growth and homeostasis (Tanida 2011). Autophagy dysregulation contributes to various diseases, including cancer, immune disorders, infectious diseases, heart disease and neurodegeneration (Gozuacik \& Kimchi 2007, Jiang et al. 2015). Previous study has reported that the level of autophagy is correlated with the sensitivity of cancer cells to chemotherapy. Autophagy inhibition could suppress the chemosensitivity of mouse embryonic fibroblasts cells to tumor necrosis factor alpha treatment (Wang et al. 2008). In addition, miRNA-93 could increase the chemotherapeutic resistance of leukemia cells by downregulating the cells' autophagy (Wu et al. 2016a). Recently, we revealed that $\mathrm{CAB}$ could inhibit the pituitary tumor growth in vitro and in vivo through the autophagic route (Leng et al. 2017). Hence, it was worth investigating whether miRNA-93 is involved in $\mathrm{CAB}$ resistance via autophagy regulation in prolactinoma.

This study reports, to our knowledge for the first time, that miRNA-93 plays crucial roles in regulating the cytotoxic effect of $\mathrm{CAB}$, which can promote the drug resistance of prolactinoma via downregulation of CAB-induced cells autophagy by directly targeting autophagy-related 7 (ATG7). Silencing of miRNA-93 expression or ATG7 overexpression can decrease the drug resistance of pituitary tumors to $\mathrm{CAB}$.

\section{Materials and methods}

\section{Cell culture and transfection}

We purchased rat prolactinoma MMQ cell lines (ATCC CRL-10609) and GH3 cell lines (ATCC CCL-82.1) from the American Type Culture Collection (ATCC). All cells were grown in Ham's F-12K (Kaighn's) medium (Gibco, Life Technologies), supplemented with $10 \%$

(C) 2019 Society for Endocrinology Published by Bioscientifica Ltd. Printed in Great Britain fetal bovine serum (Gibco) and 100 $/ \mathrm{mL}$ penicillin/ streptomycin (Gibco). We cultured cells in a $5 \% \mathrm{CO}_{2}$ atmosphere at $37^{\circ} \mathrm{C}$.

In addition, siRNA of Becn1, miRNA-93-5p inhibitor, miRNA-93-5p mimic and corresponding negative control were purchased from GenePharma (Shanghai, China) (Table 1). Hereinafter, miRNA-93 is specifically referred to as miR-93-5p in the 'Materials and methods' and 'Results' sections. The coding domain sequence of the rat Flag-Atg7 mRNA was amplified by PCR using the rat cDNA as a template and inserted into the pcDNA 3.1 vector (General Biosystems, Inc.). We performed transfection using the Lipofectamine2000 Transfection Reagent (Invitrogen) as per the manufacturer's instructions.

\section{Cell viability and colony formation assay}

The cell proliferation was assayed using the MTS-based CellTiter 96 AQueous One solution cell proliferation assay (Promega) as per the manufacturer's instructions. The absorbance was read at $490 \mathrm{~nm}$ using a plate reader (TECAN, Männedorf, Switzerland). After 48-h transfection with miRNA-93 mimics or miRNA-93 inhibitor, we plated cells $\left(5 \times 10^{3}\right)$ into six-well plates and then treated them with $100 \mu \mathrm{M}$ CAB or phosphate-buffered saline (PBS) for 2 weeks. Next, cells were stained with the Crystal Violet Staining Solution (Beyotime Biotechnology) as per the manufacturer's instructions. Furthermore, microscopy was performed using an Axiovert 200 microscope (Carl Zeiss, Oberkochen, Germany), and cell colonies were counted using PhotoShop CS6.

\section{Reverse transcription and quantitative PCR (RT-qPCR)}

The total RNA was extracted using TRIzol reagent (Invitrogen), and cDNAs were synthesized using the SuperScript III First-Strand Synthesis System for RT-qPCR (Invitrogen) (Table 2).

The RT-qPCR analysis was performed using the SYBR Green PCR Kit (Qiagen) and the 7500-Fast Real-time PCR system (Applied Biosystems). For the miRNA assay, the total RNA was extracted using the miRNeasy Mini Kit (Qiagen). Furthermore, miRNA-93 expression analysis was

Table 1 siRNA sequences.

siBecn 1

5'-CUCAGGAGAGGAGCCAUUUTT-3' 5'-AAAUGGCUCCUCUCCUGAGTT-3';

miRNA-93-5p mimic 5'-CAAAGUGCUGUUCGUGCAGGUAG-3' 5'-ACCUGCACGAACAGCACUUUGUU-3'; miRNA-93-5p inhibitor 5'-CUACCUGCACGAACAGCACUUUG-3' 
Table 2 cDNA primers.

\begin{tabular}{l} 
Primer \\
\hline Gapdh \\
Atg7 \\
miRNA-93 \\
U6
\end{tabular}

\begin{tabular}{l}
\hline Forward $\left(5^{\prime}-3^{\prime}\right)$ \\
\hline ACCCTGTTGCTGTAGCCATATTC \\
GCGGGTTCAACATGAGCATC \\
ATCAAAGTGCTGTTCGTGC \\
CTCGCTTCGGCAGCACA
\end{tabular}

\begin{tabular}{l} 
Reverse $\left(5^{\prime}-3^{\prime}\right)$ \\
\hline ACCCTGTTGCTGTAGCCATATTC \\
TACTCCTGAGCTGTGGTTGC \\
GTGCAGGGTCCGAGGT \\
AACGCTTCACGAATTTGCGT
\end{tabular}

performed using the miScript PCR System (Qiagen) as per the manufacturer's instructions. The $\Delta \Delta \mathrm{CT}$ value was used to evaluate the relative miRNA-93 expression using U6 as an endogenous control.

\section{Western blotting}

The following antibodies were sued in this study: ATG7 antibody (\#8558; Cell Signaling Technology), LC3 antibody (\#3868; Cell Signaling Technology), BECN1 antibody (\#3738; Cell Signaling Technology), tubulin antibody (11224-1-AP; Proteintech Group, Chicago, IL, USA); caspase-8 antibody (13423-1-AP; Proteintech Group); antirabbit IgG, horseradish peroxidase (HRP)-linked antibody (\#7074; Cell Signaling Technology) and anti-mouse IgG, HRP-linked antibody (\#7076; Cell Signaling Technology). Cells were lysed with the radioimmunoprecipitation assay (RIPA) buffer (P0013C; Beyotime Biotechnology), and the total protein concentration was measured using the Bicinchoninic Acid Protein Assay Kit (PA115; Tiangen Biotech). In addition, proteins from whole-cell extracts were resolved using denaturing SDS-PAGE and analyzed by Western blotting. Subsequently, equal amounts of protein were separated using SDS-PAGE and transferred to polyvinylidene fluoride membranes (Millipore). Protein bands were detected with Pierce ECL chemiluminescence reagents (Thermo Fisher) and exposed on the ImageQuant LAS 4000 (Japan) system. Immunoblots shown in figures were derived from three independent experiments. Furthermore, intensities of protein bands were quantified by densitometry using the ImageJ software (http://imagej. nih.gov/ij/).

\section{Immunocytochemistry}

Cells were grown to $50 \%$ confluence on glass coverslips in a 12-well tissue culture plate and then treated with the appropriate drug at the indicated concentrations for the stated duration. Next, cells were washed thrice with sterile PBS (8.4 mM Na $\mathrm{HPO}_{4}, 1.5 \mathrm{mM} \mathrm{KH} \mathrm{PO}_{4}, 136.9 \mathrm{mM}$ $\mathrm{NaCl}$ and $2.7 \mathrm{mM} \mathrm{KCl}, \mathrm{pH} 7.2$ ), fixed and permeabilized in acetone for $10 \mathrm{~min}$ and blocked with $3 \%$ bovine serum albumin (ST023; Beyotime Biotechnology) for $2 \mathrm{~h}$.
Primary immunostaining with the LC3 antibody (L7543; Sigma-Aldrich) was performed at room temperature overnight, followed by immunostaining with Alexa Fluor 488-conjugated secondary antibody (A-11008; Invitrogen). Subsequently, the nucleus was counterstained with 4',6-diamidino-2-phenylindole (28718-90-3; SigmaAldrich) for $15 \mathrm{~min}$ at room temperature. Staining was visualized and photographed using an LSM710 laser scanning confocal microscope with an oil immersion lens (Carl Zeiss). Cell images were independently validated by performing cell counting on five different magnification fields per image: LC3 puncta per cell=number of LC3 puncta/total number of cells.

\section{Reporter constructs and luciferase assays}

We prepared the $3^{\prime}$-UTR luciferase reporter construct by cloning the human mRNA 3 '-UTR sequence into the pMIR-Report construct (Ambion, Austin, TX, USA). The Atg7 3'-UTR fragment (from 1236 to 1643) was amplified and cloned into the luciferase reporter via SacII and EcoRI sites. Next, oligonucleotides bearing the designed sequence were inserted at the same site to obtain the Atg7 3'-UTR point mutation. Luciferase reporter assays were performed as reported previously (Wu et al. 2013). Briefly, MMQ and GH3 cells plated in a 24-well plate were cotransfected with $100 \mathrm{nM}$ single-stranded miRNA mimics, 25-ng firefly luciferase reporter comprising Atg7 3'-UTR and 6-ng pRL-TK (Promega) using the JetPRIME reagent (Polyplus-transfection). After 36-h transfection, cells were collected and analyzed using the Dual-Luciferase Reporter Assay System (Promega).

\section{Immunohistochemical staining of ATG7 in the pituitary tumor tissue}

Antigens were retrieved from formaldehyde-fixed, paraffin-embedded (FFPE) tumor tissue sections from six DA-resistant and six DA-sensitive human prolactinomas by boiling in sodium citrate buffer ( $\mathrm{pH}$ 6.0) for $30 \mathrm{~min}$. The $5 \mu \mathrm{m}$ tissue sections were dehydrated and subjected to peroxidase blocking. Next, immunohistochemical staining was performed by incubating tissue sections using 
rabbit anti-human ATG7 primary antibody (ab133528; Abcam) overnight at $4^{\circ} \mathrm{C}$ with gentle shaking, followed by incubation with goat anti-rabbit HRP secondary antibody for $1 \mathrm{~h}$ at room temperature. Subsequently, the sections were exposed to 3,3'-diaminobenzidine substrate (dissolved in the Dako substrate buffer, Cat. No. 760-500; Roche), followed by Gill's haematoxylin counterstaining and standard dehydration treatment. The staining images were obtained using an Axiovert 200 microscope (Carl Zeiss).

Further, the immunohistochemical staining was evaluated in a semiquantitative manner by two senior neuropathologists blinded to the clinical parameters. Cytoplasm staining was considered positive. We selected 10 high-power fields $(400 \times)$ within the tumor showing cytoplasm staining to evaluate the ATG7 expression level. Furthermore, the percentage of the staining extent was calculated according to the percentages of positively stained areas of tumor cells in relation to the whole carcinoma area.

\section{Tumor formation assay}

Female athymic nude mice were purchased from the SLAC (Shanghai Slack Laboratory Animal Co., Ltd., Shanghai, China), which were kept under specific pathogen-free conditions. Sixteen female nude mice were divided into four groups. Subsequently, MMQ cells transfected with miRNA-93 inhibitor or miRNA-NC for $24 \mathrm{~h}$, trypsinized and then resuspended in PBS; the transfection effects were tested using RT-qPCR, followed by subcutaneously injecting 6-week-old nude mice with $10^{6}$ cells in $100 \mu \mathrm{L}$ PBS per injection. When the tumor volume reached $25 \mathrm{~mm}^{3}$, the tumors were treated with $200 \mathrm{nM}$ miRNA-93 inhibitor or miRNA-NC through local injection of a xenograft tumor at multiple sites. This treatment was performed once every 2 days for 16 days. For drug administration, animals were intraperitoneally injected with $100 \mu \mathrm{L}$ of vehicle control $(0.1 \%$ dimethyl sulfoxide in $0.9 \%$ saline) or $0.5-\mathrm{mg} / \mathrm{kg}$ CAB daily. After 16 days, all animals were killed and weighed simultaneously. Notably, no animal died before the end of the experiment. The tumor size was measured by a Vernier caliper every 3 days and calculated as $\left(\right.$ length $\times$ width $\left.^{2}\right) / 2$. All procedures in this study were approved by the Laboratory Animal Ethics Committee of Wenzhou Medical University (permission number: wydw2014-0026) and performed in accordance with the National Institutes of Health Guide for the Care and Use of Laboratory Animals. The total protein of the tumor tissue was extracted with RIPA buffer (Beyotime Biotechnology) and analyzed by Western blotting as described previously.

(C) 2019 Society for Endocrinology Published by Bioscientifica Ltd. Printed in Great Britain

\section{Cytokine ELISAs}

The PRL level in the plasma of tumor-bearing nude mice was measured using a commercially available ELISA kit, as per the manufacturer's instructions (ExCell Biology, China). The blood of tumor-bearing nude mice was collected from the angular vein. The minimal detectable concentration of PRL was $4 \mathrm{pg} / \mathrm{mL}$. The inter-assay and intra-assay coefficients of variation were $<10 \%$. All independent experiments were performed in triplicate.

\section{Transmission electron microscopy (TEM)}

Samples were tested at the Electron Microscopy Center in Shanghai Jiaotong University. The tumor tissue obtained from the MMQ xenograft tumors of mice were fixed with $2.5 \%$ glutaraldehyde overnight and post-fixed with $1 \%$ osmium tetroxide $(\mathrm{pH} \mathrm{7.4)}$ ) for $2 \mathrm{~h}$ at room temperature. The pellets were then dehydrated in graded ethanol series, infiltrated and embedded in Spurr's resin (01916-1; Polysciences). Samples were then polymerized for $48 \mathrm{~h}$ at $60^{\circ} \mathrm{C}$, cut into 60 -nm-thick sections on LKB-I microtome (LKB, Sweden), positioned on 200-mesh grids (22896-1; Polysciences) and stained with uranyl acetate and lead citrate. TEM was performed on PHILIPS CM120 TEM at an accelerating voltage of $120 \mathrm{kV}$. Furthermore, images were acquired using Gatan type UltraScan 4000SP CCD Camera connected to the transmission electron microscope.

\section{Statistical analysis}

All statistical analyses were performed using the GraphPad Prism, version 5 (GraphPad Software). All continuous variables are expressed as mean \pm standard error of the mean. Student's unpaired, two-tailed $t$-tests with a 95\% CI were used to analyze data involving direct comparison of an experimental group with a control group. A two-tailed $P$-value test was used with $P<0.05$ considered statistically significant.

\section{Results}

\section{miRNA-93 inhibited CAB efficiency in vitro}

To elucidate the biological function of miRNA-93, we elevated the total miRNA-93 level by transfecting miRNA-93 mimic to MMQ and GH3 cells and then subjecting these cells to the subsequent experiment. As shown in Fig. 1, MMQ and GH3 cells transfected with miRNA-93 mimic exhibited upregulated miRNA-93 expression, as assessed 


\begin{tabular}{l|l|l|l|l|}
$\begin{array}{l}\text { Journal of } \\
\text { Endocrinology }\end{array}$ & Z Wu, L Cai, J Lu et al. & $\begin{array}{c}\text { miR-93 mediates effect of CAB } \\
\text { by targeting ATG7 }\end{array}$ & $\mathbf{2 4 0 : 1}$ & $\mathbf{5}$ \\
\hline
\end{tabular}

A

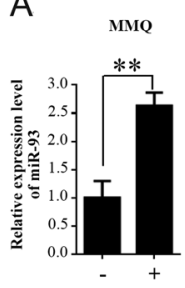

miR-93 mimic

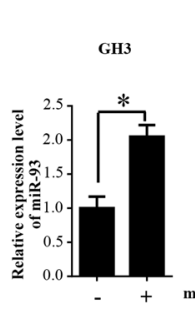

miR-93 mimic
B

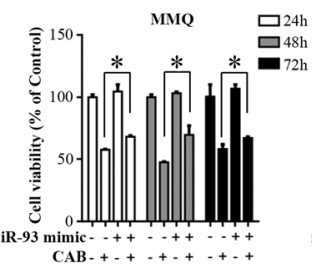

C

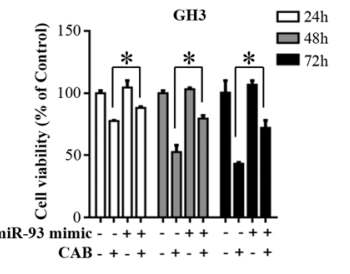

D

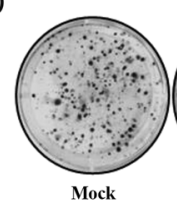

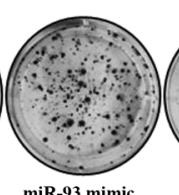

miR-93 mimic

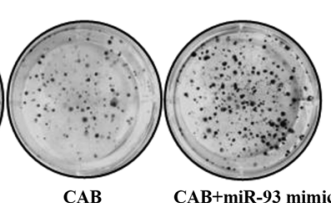

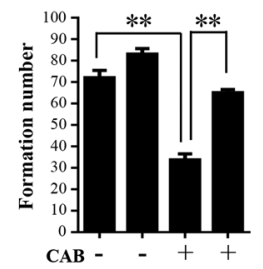

miR-93 mimic - +-+

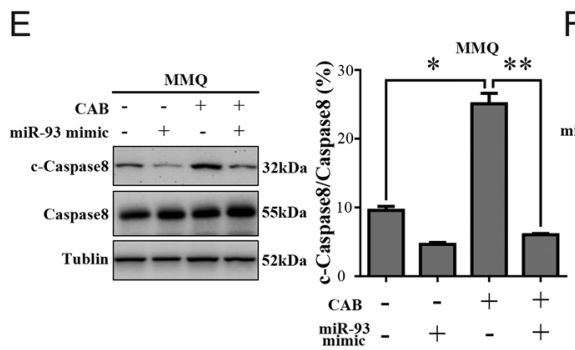

$\mathrm{F}$

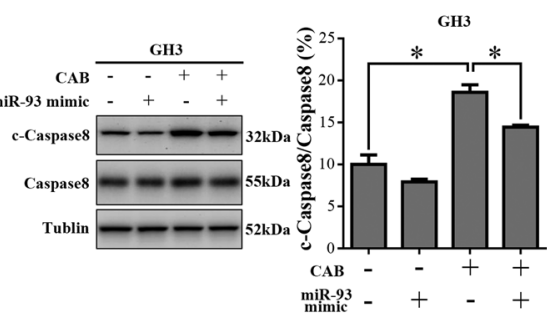

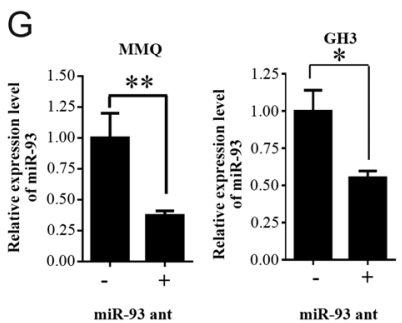

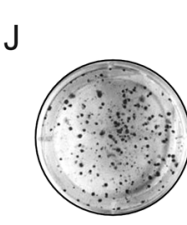

Mock

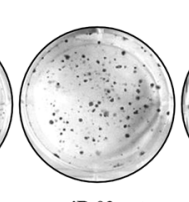

miR-93 ant

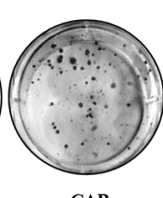

$\mathrm{CAB}$

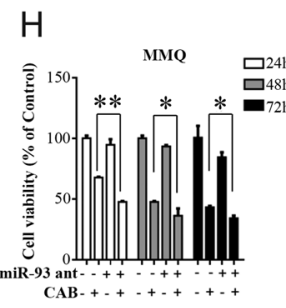

$\mathrm{K}$
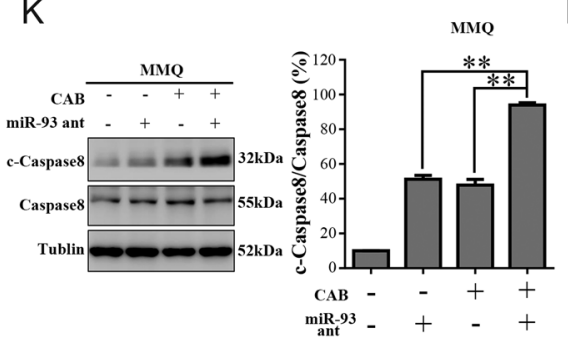

L
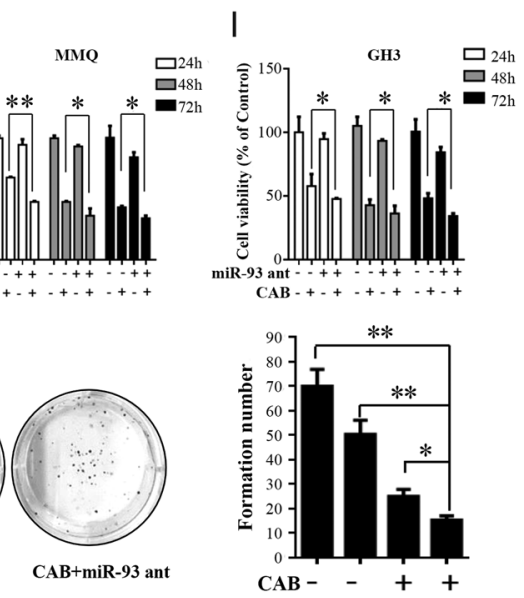

miR-93 ant $-+\quad+$

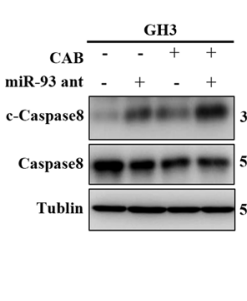

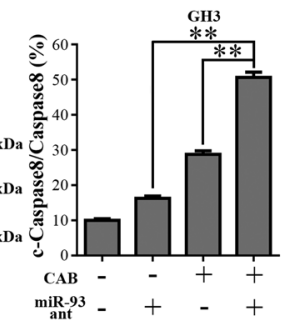

\section{Figure 1}

miRNA-93 decreased CAB $(100 \mu \mathrm{M}$ in GH3 and $50 \mu \mathrm{M}$ in MMQ)-induced cell death. (A) The effect of miRNA-93 mimic. The MMQ and GH3 cells were transfected with miRNA-93 mimic or miRNA-NC for $48 \mathrm{~h}$ and subjected to quantitative PCR analysis of miRNA-93 expression. (B and C) miRNA-93 mimic could downregulate CAB-induced cell death in MMQ and GH3 cells. (D) The clone formation assays of $\mathrm{GH} 3$; the $\mathrm{CAB}$ treatment resulted in the inhibition of cell colonies, but miRNA-93 mimic could inhibit CAB-induced cell death. (E and F) The immunoblot analysis of the apoptosis-related protein. The cleaved caspase-8 expression in $C A B$ groups increased in MMQ and GH3 cells, and miRNA-93 mimic could inhibit this effect. (G) The effect of miRNA-93 inhibitor. The MMQ and GH3 cells were transfected with miRNA-93 inhibitor or miRNA-NC for $48 \mathrm{~h}$ and subjected to quantitative PCR analysis of miRNA-93 expression. ( $\mathrm{H}$ and $\mathrm{I})$ The miRNA-93 inhibitor could promote $\mathrm{CAB}$-induced cell death in MMQ and GH3 cells. (J) In the clone formation assays, the miRNA-93 inhibitor could promote CAB-induced cell death. ( $K$ and $L$ ) The immunoblot analysis of the apoptosis-related protein; the cleaved caspase-8 expression in $\mathrm{CAB}$ groups increased in $\mathrm{MMQ}$ and GH3 cells, and miRNA-93 inhibitor could enhance this effect. ${ }^{*} P<0.01 ;{ }^{*} P<0.05$. using RT-qPCR ( $P<0.05$; Fig. 1A). miRNA-93 expression significantly increased by $167 \%$ in MMQ cells and by 109\% in GH3 cells after miRNA-93 mimic transfection. Subsequently, the cell viability assays revealed that miRNA-93 mimic could decrease CAB-induced cell death in MMQ and GH3 cells ( $P<0.05$; Fig. 1B and C). Compared with $\mathrm{CAB}$ treatment, the cell viability increased by $22.1 \%$ in MMQ cells and $27.0 \%$ in GH3 cells after miRNA-93 mimic $+\mathrm{CAB}$ treatment at $48 \mathrm{~h}$. Meanwhile, similar results were obtained from the plate clone formation assays in 
GH3 cells, which were treated with miRNA-93 mimic and $\mathrm{CAB}$ for $48 \mathrm{~h}$, determined by the formation count $(P<0.05$; Fig. 1D). The formation number was $72.0 \pm 5.1$ in the control group, $83.0 \pm 6.7$ in the miRNA-93 mimic group, $35.0 \pm 7.2$ in the $\mathrm{CAB}$ group and $65.0 \pm 3.4$ in the miRNA-93 mimic and CAB group. In addition, after 48-h $\mathrm{CAB}$ treatment, the expression of the cleaved caspase- 8 protein in MMQ and GH3 cells increased. However, miRNA-93 mimic inhibited the CAB effect $(P<0.05$; Fig. $1 \mathrm{E}$ and $\mathrm{F}$ ). Compared with the $\mathrm{CAB}$ treatment alone, the cleaved caspase- 8 expression decreased by $75.88 \%$ in MMQ and by $22.32 \%$ in GH3 cells after CAB + miRNA-93 mimic treatment.

Conversely, miRNA-93 inhibitor significantly reduced the miRNA-93 expression level in MMQ and GH3 cells $(P<0.05$; Fig. 1G). The miRNA-93 expression decreased by $64 \%$ in MMQ and by $45 \%$ in GH3 cells after miRNA-93 inhibitor transfection. Subsequently, as shown in the cell viability assays, after being treated with $\mathrm{CAB}$ and miRNA-93 inhibitor, the cell viability decreased by $17.08 \%$ in MMQ cells and by $10.77 \%$ in GH3 cells after $48 \mathrm{~h}$ compared with that the cells treated with $\mathrm{CAB}(P<0.05$; Fig. $1 \mathrm{H}$ and $\mathrm{I}$ ), suggesting that downregulation of the miRNA-93 expression could promote the CAB effect. In formation assays, downregulation of the miRNA-93 expression enhanced the cytotoxic effects of $\mathrm{CAB}$ in GH3 cells $(P<0.05$; Fig. 1J). The formation number was $70.0 \pm 9.4$ in the control group, $50.0 \pm 7.2$ in the miRNA-93 inhibitor group, $25.0 \pm 3.2$ in the $\mathrm{CAB}$ group and $17.0 \pm 1.2$ in the miRNA-93 inhibitor $+\mathrm{CAB}$ group. Compared with the $\mathrm{CAB}$ treatment alone, the cleaved caspase- 8 expression increased by $46.76 \%$ in MMQ and by $23.70 \%$ in GH3 cells after $\mathrm{CAB}+$ miRNA-93 inhibitor treatment (Fig. $1 \mathrm{~K}$ and $\mathrm{L}$ ).

\section{miRNA-93 inhibited CAB-induced autophagy in pituitary tumor cell lines}

As described previously, $\mathrm{CAB}$ inhibits the pituitary tumor growth in vivo and in vitro by inducing autophagic cell death (Lin et al. 2015). Interestingly, in Western blotting, we found that serum starvation could promote the LC3-II protein expression, which is the marker protein of autophagy. However, the miRNA-93 overexpression in MMQ cells (Fig. 2A) and GH3 cells (Fig. 2B) could inhibit the LC3-II protein expression and reverse the serum deprivation-induced autophagy. Next, we tested the LC3-II protein level in MMQ cells and GH3 cells, which were cotreated with $\mathrm{CAB}$ and miRNA-93 mimics, to further confirm whether miRNA-93 was involved in the CAB-induced autophagy. The findings revealed that $\mathrm{CAB}$ was related to the induction of autophagy in MMQ and GH3 cells; the LC3-II expression increased by 9.1-fold in MMQ and by 2.3-fold in GH3 cells after the CAB treatment. Conversely, the increasing amplitude in the $\mathrm{CAB}+$ miRNA-93 mimics group was less than that in the $C A B$ group (Fig. 2C). Moreover, the LC3-II protein level was markedly upregulated in $\mathrm{MMQ}$ and $\mathrm{GH} 3$ cells after treatment with $\mathrm{CAB}+$ miRNA-93 inhibitor (Fig. 2D). In addition, the immunochemistry assay revealed that miRNA-93 mimics could downregulate the CAB-induced LC3 puncta accumulation, on the contrary, miRNA-93 inhibitor could upregulate the CAB-induced LC3 puncta accumulation in MMQ and GH3 cells $(P<0.05$, Fig. $2 \mathrm{E}$ and F). Overall, these findings indicated that miRNA-93 could inhibit CABinduced autophagy and decrease the LC3-II expression.

\section{Knocking-down of Becn1 reversed the effect of miRNA-93 inhibitor}

Previous studies have reported that BECN1, a crucial autophagy-related gene, is an autophagy regulator and tumor suppressor (Cicchini et al. 2014). Thus, to further investigate whether the miRNA-93-mediated downregulation of autophagy could affect CAB-induced autophagic cell death, we transfected MMQ and GH3 cells with a siRNA to knockdown the Becn1 expression. The transfection efficiency was confirmed by Western blotting, and knocking downing the BECN1 expression could partially inhibit the apoptosis-related cleaved caspase-8 protein level (Fig. 3A). In cell viability assays, the miRNA-93 inhibitor and CAB treatment could inhibit the proliferation of MMQ and GH3 cells, and the miRNA-93 inhibitor could enhance the cytotoxic effect of $\mathrm{CAB}$ in MMQ and GH3 cells ( $P<0.05$; Fig. 3B and C). Nonetheless, knocking down the BECN1 expression could partially suppress the miRNA-93 inhibitor's enhancement effect on $\mathrm{CAB}(P<0.05)$. Compared with the CAB + miRNA-93 inhibitor treatment, the cell viability increased by $13.35 \%$ in MMQ cells and by $29.85 \%$ in GH3 cells after CAB + miRNA-93 inhibitor + siBecn 1 treatment. There was no difference between $\mathrm{CAB}$ treatment and $\mathrm{CAB}+$ miRNA-93 inhibitor + siBecn 1 treatment. Furthermore, Western blotting revealed that the miRNA-93 inhibitor could promote the expression of the cleaved caspase- 8 , which was considered as the CAB-induced apoptosis-related protein. However, silencing the BECN1 expression partially abolished this effect in MMQ cells $(P<0.05$; Fig. 3D) and GH3 cells $(P<0.05$; Fig. 3E). These findings suggested that miRNA-93 decreased the CAB-induced apoptosis in MMQ and GH3 cells by downregulating autophagy. 
A
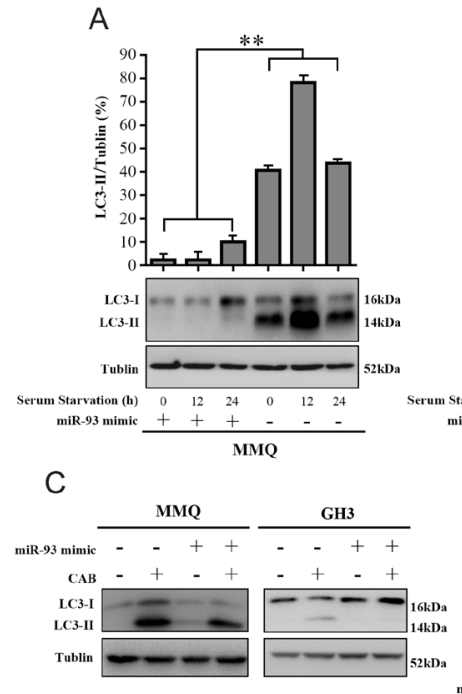

D

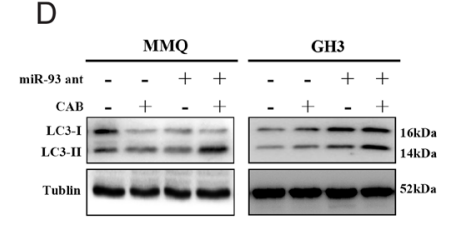

E

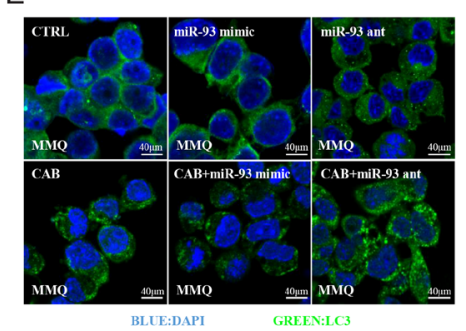

F

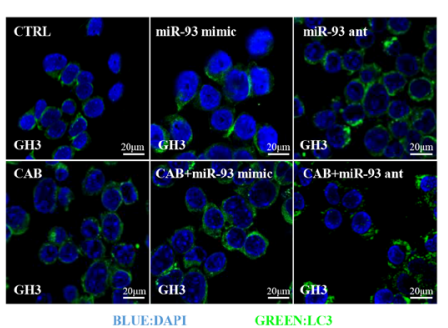

B
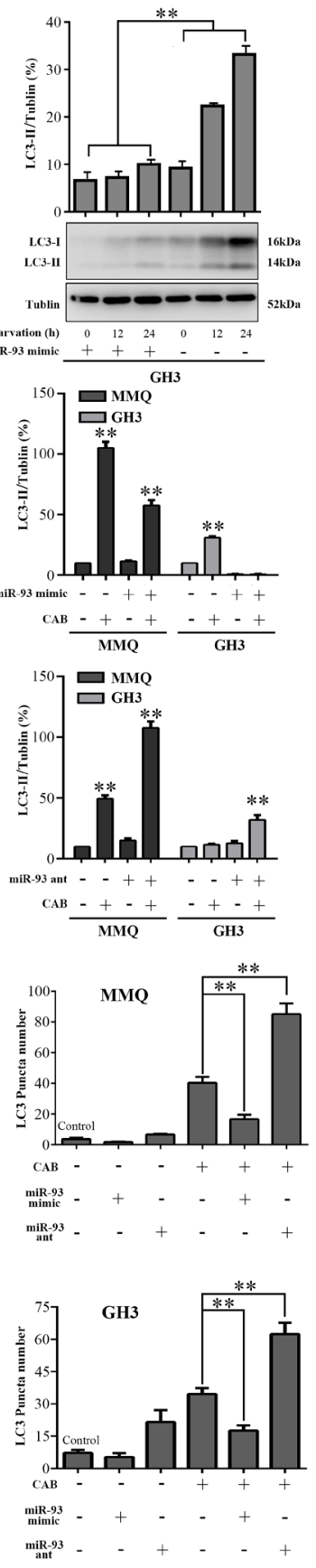

Figure 2

miRNA-93 inhibited the CAB-induced autophagy in pituitary tumor cell lines. (A and B) The immunoblot analysis of LC3-I and LC3-II in MMQ and GH3 cells treated as described. miRNA-93 in MMQ cells (A) and GH3 cells (B) could inhibit the LC3-II protein expression and reverse the serum deprivationinduced autophagy. ( $C$ and D) CAB could activate autophagy, and the miRNA-93 overexpression could downregulate the LC3-II expression and inhibit the CAB-induced autophagy in MMQ and GH3 cells (C). However, the downregulation of miRNA-93 expression could enhance this effect (D). ( $E$ and F) miRNA-93 could reverse the CAB-induced LC3 puncta accumulation, on the contrary, the downregulation of miRNA-93 expression could promote the CAB-induced LC3 puncta in MMQ and GH3 cells. ** $P<0.01 ; * P<0.05$.

\section{ATG7 was the direct target of miRNA-93}

We used the bioinformatics database (TargetScan; http:// www.targetscan.org/vert_71/) to predict the targets of miRNA-93. Atg7, which is a hypothetical target of miRNA-93, provoked our interest. We previously reported that silencing the ATG7 expression can reverse the CAB efficiency in pituitary tumors (Lin et al. 2015). Recently, a study reported that ATG7 acts as a crucial member of the autophagic-related protein family, which has a pleiotropic function in the tumor progression (Xiong 2015). As described earlier that miRNA-93 was involved in regulating the CAB-induced autophagy, we further investigated the correlation between Atg7 and miRNA-93. The findings indicated that putative miRNA-93-binding sites were located in the 3'-UTR of the Atg7 transcript. We performed luciferase reporter assays in GH3 and MMQ cells to confirm whether miRNA-93 affected the Atg7 expression. In addition, we constructed the Atg7 WT and Atg7 mutant luciferase reporter plasmid (Fig. 4A). The administration of single-stranded oligonucleotides (mimics), which directly promoted miRNA-93, could significantly downregulate the expression of a luciferase reporter containing the $3^{\prime}$-UTR sequence from $A \operatorname{tg} 7$ in MMQ cells $(P<0.05$; Fig. $4 \mathrm{~B})$ and $\mathrm{GH} 3$ cells $(P<0.05$; Fig. 4C). No change was observed in the luciferase activity in the mutant luciferase vector. Subsequently, we explored whether the endogenous Atg7 in GH3 and MMQ cells were similarly regulated by miRNA-93. GH3 and MMQ cells were transfected with miRNA-93 mimics, and the Atg7 mRNA level in MMQ cells $(P<0.05$; Fig. 4D) and GH3 cells $(P<0.05$; Fig. $4 \mathrm{E})$ were measured by RT-qPCR protein levels were then examined by Western blotting $(P<0.01 ;$ Fig. 4F). The ATG7 level was substantially downregulated by miRNA-93 mimics. Overall, these findings demonstrated that ATG7 was the direct target of miRNA-93.

In addition, we further tested the ATG7 expression in human prolactinoma. Immunohistochemistry (IHC) was performed in six DA-sensitive human prolactinoma tissues and six DA-resistant human prolactinoma tissues. As shown in Fig. 4G, the ATG7 protein was primarily localized to the cytoplasm of tumor cells. The ATG7 protein expression increased in drug-sensitive human prolactinoma compared with that in drug-resistant human prolactinoma. The average IHC score of the ATG7 expression is shown in Fig. $4 \mathrm{G}(64.17 \pm 5.540 ; 95 \% \mathrm{CI}$ : 49.92-78.40 vs $30.83 \pm 5.972$, 95\% CI: 15.47-46.19; $P=0.0014)$. 
A
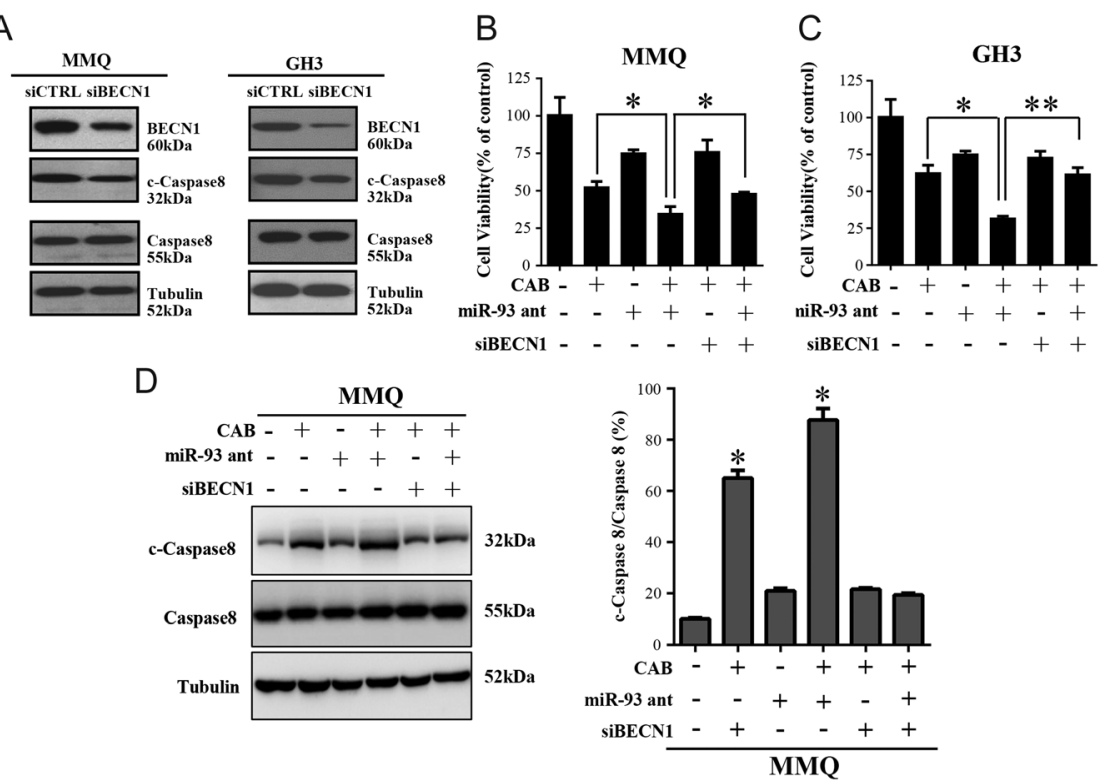

$E$
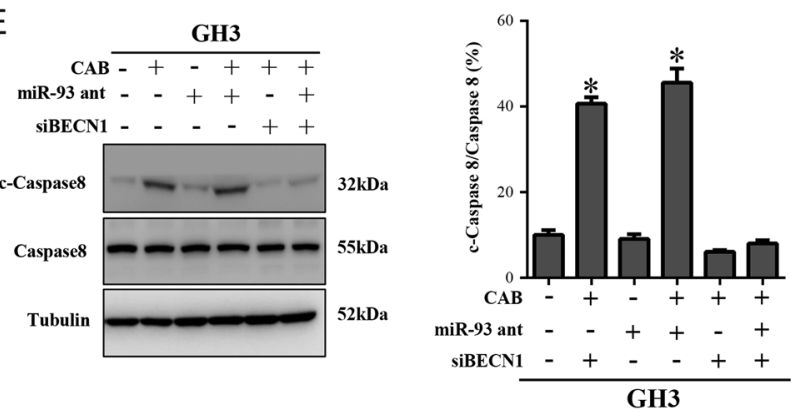

Figure 3
Silencing the Becn1 expression could reverse the
effect of the miRNA-93 inhibitor. (A) The effect of
the BECN1 knockdown by siRNA silencing. The
cells were transfected with siControl or siBecn1
for $48 \mathrm{~h}$ and subjected to immunoblot analysis of
the HMGB2 expression. Knocking down the
BECN1 expression could partially inhibit the
cleaved caspase-8 protein expression. (B and C)
BECN1 downregulation could reverse the
miRNA-93 inhibitor's enhancement effect on CAB
and promote the cell viability in MMQ and GH3
cells. (D and E) In the immunoblot analysis, the
cleaved caspase-8 expression in CAB groups
increased in MMQ and GH3 cells, and the
miRNA-93 inhibitor could enhance this effect.
However, silencing the BECN1 expression could
partially abolish this effect. $* * P<0.01$; $* P<0.05$.

\section{Overexpression of ATG7 reversed the effect of miRNA-93 in pituitary tumor}

We performed rescue experiments to evaluate whether ATG7 was responsible for the functional effects of miRNA-93 in pituitary tumor cells. First, MMQ and GH3 cells were transfected with the miRNA-93 mimic and Flag-tagged Atg7 vector. After 48-h transfection, Western blotting was used to confirm the transfection efficiency (Fig. 5A). As shown in Fig. 5B and C, compared with the CAB+miRNA-93 mimics treatment, the cell viability decreased by $33.33 \%$ in MMQ cells and by $38.89 \%$ in GH3 cells after $\mathrm{CAB}+$ miRNA-93 mimics+Flag-Atg7 treatment. On the contrary, there was no difference between $\mathrm{CAB}$ treatment and $\mathrm{CAB}+$ miRNA-93 mimics + Flag-Atg7 treatment. These results indicated that the Flag-Atg 7 overexpression could reverse the inhibitory effect of miRNA-93 mimic to the CAB-induced cytotoxic effect in MMQ cells (Fig. 5B) and GH3 cells (Fig. 5C). Meanwhile, we used Western blotting to measure the LC3-II protein and cleaved caspase-8 protein expression in MMQ cells $(P<0.01$; Fig. 5D) and GH3 cells $(P<0.01 ;$ Fig. $5 \mathrm{E})$. The results revealed that overexpressed Flag-Atg7 reversed the miRNA-93-mediated inhibitory effect on the CAB-induced autophagy and apoptosis in pituitary tumor cells. Overall, these data clearly demonstrated that miRNA-93 inhibited the CAB-induced cell death and autophagy by targeting Atg7.

\section{miRNA-93 inhibitor enhanced CAB efficiency in vivo}

MMQ cells transfected with the miRNA-93 inhibitor or miRNA-NC were subcutaneously injected into nude mice to determine whether miRNA-93 affected CAB efficiency in vivo. As shown in Fig. $6 \mathrm{~A}$, the CAB treatment $(0.5 \mathrm{mg} / \mathrm{kg}$, twice a week) could inhibit the tumor growth in vivo, and the miRNA-93 inhibitor could enhance the therapeutic effectiveness of $C A B$. We found that the tumor growth $(P<0.05$; Fig. 6B) and weight $(P<0.01$; Fig. 6C) in the $\mathrm{CAB}+$ miRNA-93 inhibitor group were markedly inhibited compared with those in other three groups. The tumor volumes were $148.61 \pm 13.56 \mathrm{~mm}^{3}$ in the miRNA-93 inhibitor group, $140.41 \pm 12.74 \mathrm{~mm}^{3}$ in the $\mathrm{CAB}$ group and $79.33 \pm 5.81 \mathrm{~mm}^{3}$ in the $\mathrm{CAB}+$ miRNA-93 inhibitor group, respectively. Furthermore, tumor weights were $0.58 \pm 0.09 \mathrm{~g}$ in the miRNA-93 inhibitor group, $0.45 \pm 0.05 \mathrm{~g}$ in the $\mathrm{CAB}$ group and $0.11 \pm 0.03 \mathrm{~g}$ in the $\mathrm{CAB}+$ miRNA-93 
'WTATG7 3' UTR 5 ' ...CUAUGUGGUUUAAGAGCACUUUA......

Position 1236-1643

miR-93-5p 3'

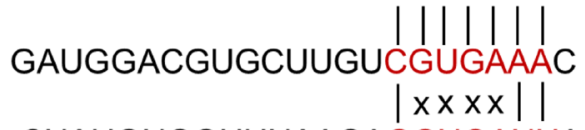

MUT ATG7 3' UTR 5' ...CUAUGUGGUUUAAGAGGUGAUUA......

B
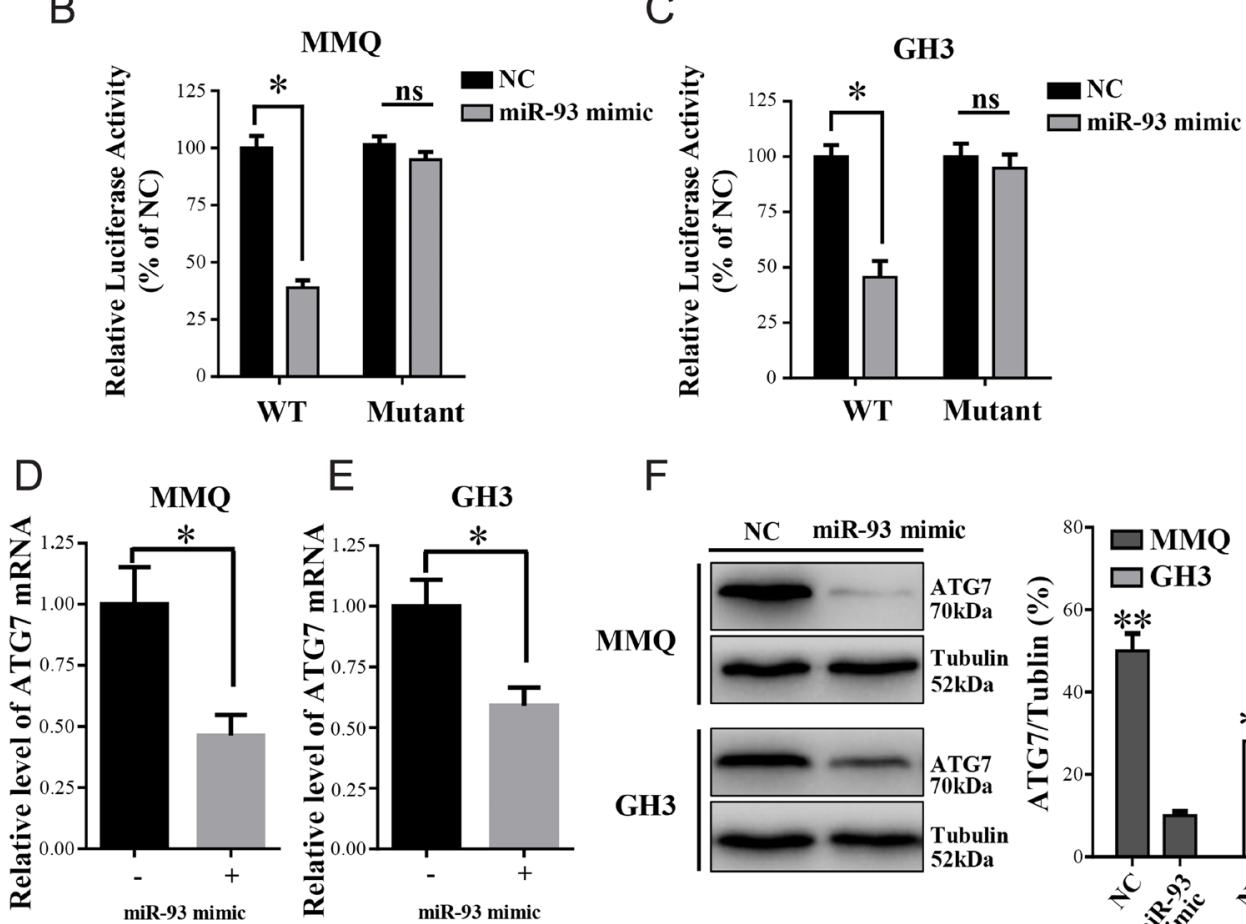

E

F
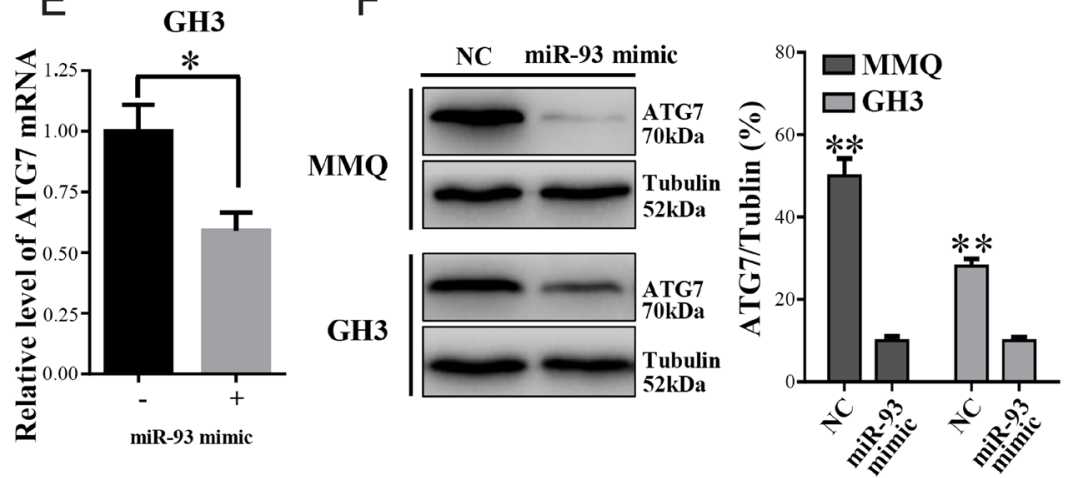

G
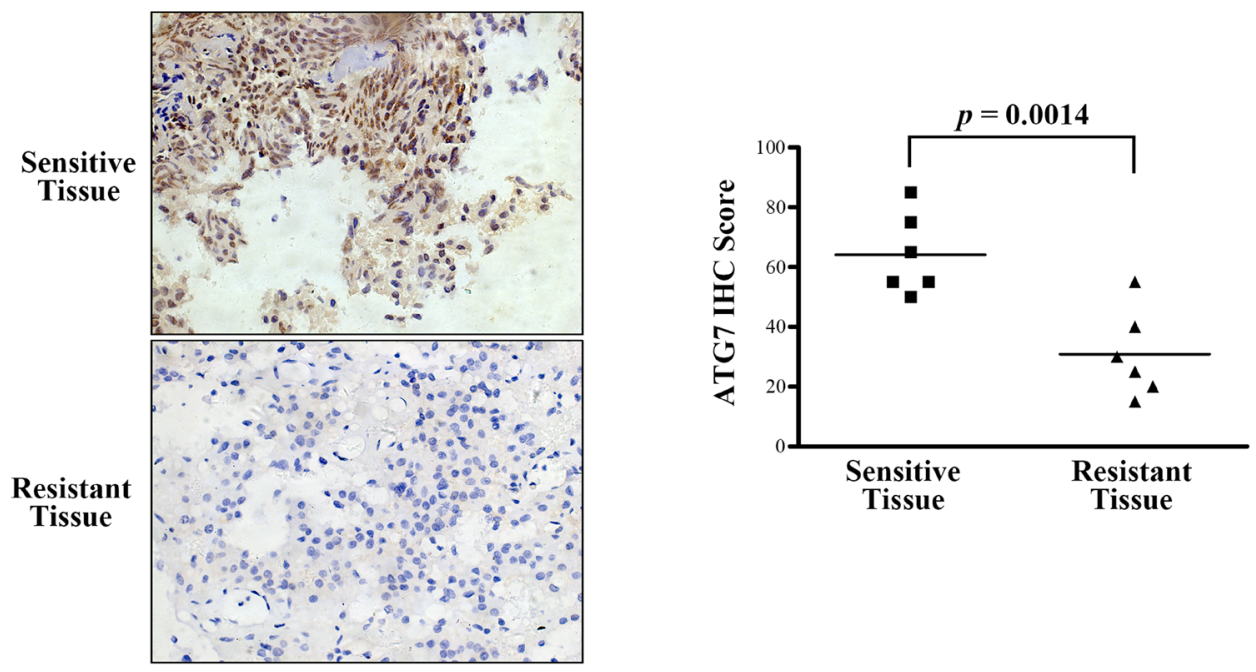

Figure 4

Atg7 was a direct target of miRNA-93. (A) A sequence alignment of miRNA-93 and its target sites in 3'-UTR of Atg7, which was downloaded from the bioinformatics database (TargetScan; http://www.targetscan.org/vert_71/). (B and C) The analysis of the luciferase activity. The normalized luciferase activity of mimics NC was set as relative luciferase activity in MMQ (B) and GH3 (C) cells. (D, E and F) The effects of miRNA-93 mimics on the endogenous Atg7 levels. After 48-h transfection with miRNA-93 mimics, MMQ cells and GH3 cells were analyzed by qPCR analysis and Western blotting, respectively. NC, normal control. (G) IHC was performed in six DA-sensitive human prolactinoma and six DA-resistant human prolactinoma. The ATG7 protein expression was higher in DA-sensitive human prolactinoma compared with that in DA-resistant human prolactinoma $(P=0.0013)$. $* * P<0.01$; $* P<0.05$. 
A

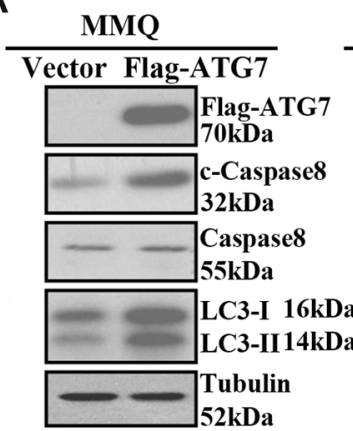

GH3

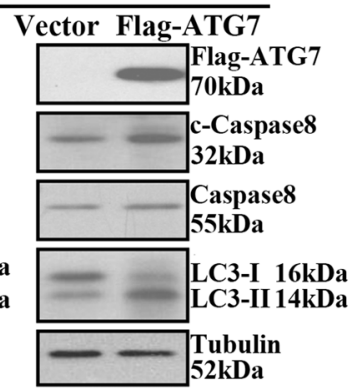

D

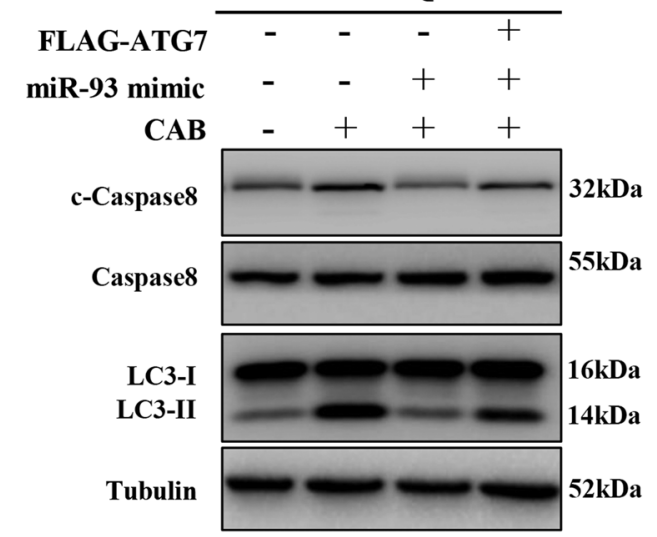

$E$

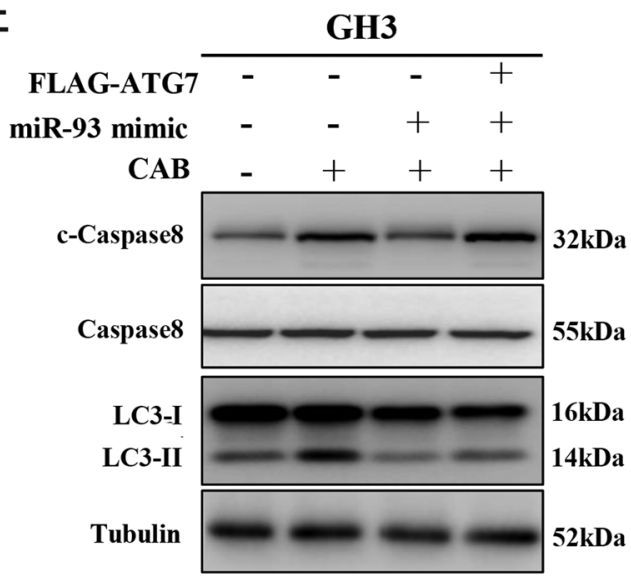

B
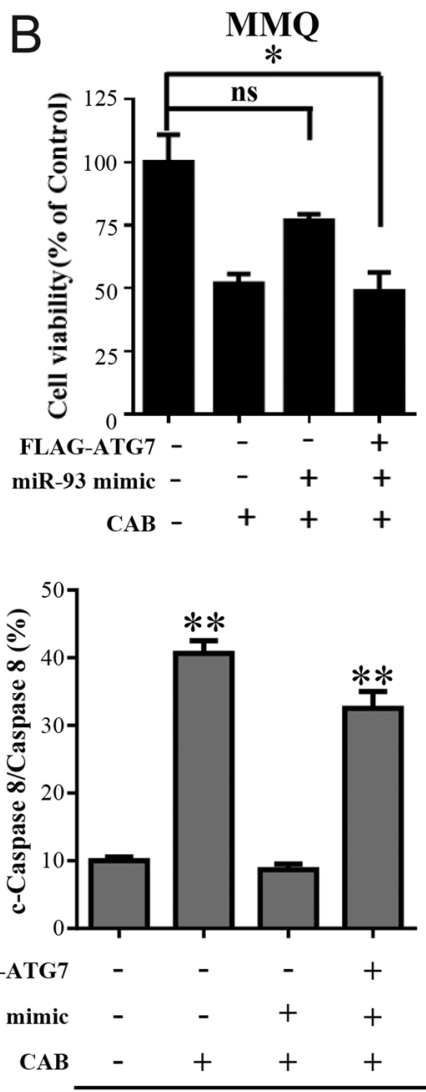

MMQ

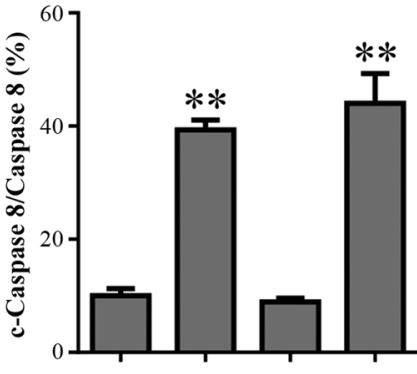

FLAG-ATG 7

miR-93 mimic

CAB

$$
\frac{\mathrm{AB}-\mathrm{+}}{\mathrm{GH} 3}
$$

C GH3

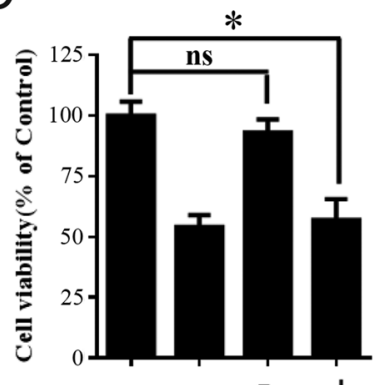

FLAG-ATG7 - - - +

miR-93 mimic - $\quad+\quad+$

САB - +++

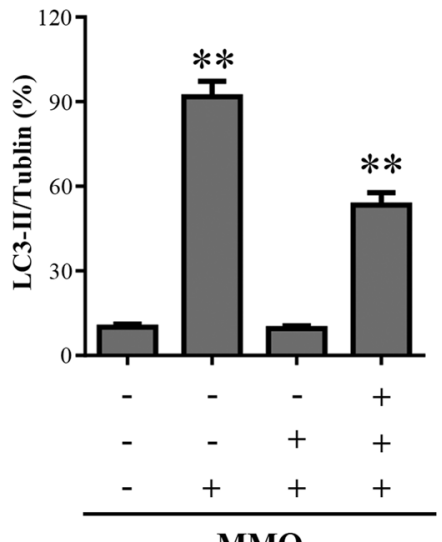

MMQ

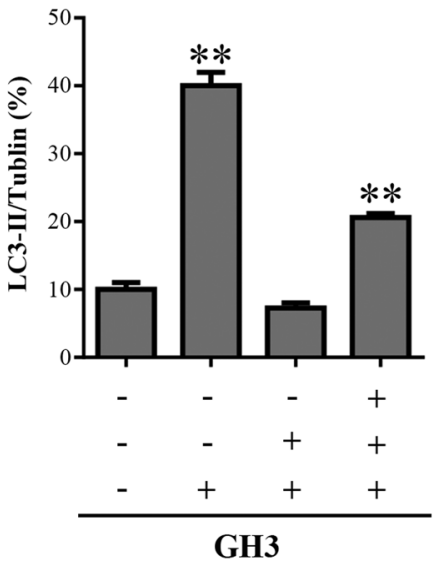

Figure 5

miRNA-93 regulates the CAB-induced autophagy through ATG7. (A) The Flag-ATG7 protein expression level was determined in rat pituitary tumor cells transfected with or without a Flag-Atg7 overexpression plasmid by Western blotting. The Flag-ATG7 overexpression could promote the cleaved caspase-8 and LC3-II protein expression. (B and C) The ATG7 overexpression could reverse the miRNA-93-mediated inhibitory effect on the CAB-induced cell apoptosis in pituitary tumor cells. (D and E) The ATG7 overexpression could promote the expression of cleaved caspase-8 or LC3-II and enhance the cytotoxic effect of CAB in MMQ and GH3 cells, which were inhibited by miRNA-93 mimic. ${ }^{* * P} P<0.01 ; * P<0.05$.

inhibitor group, respectively. In addition, we measured the PRL level in the serum of nude mice. We found that the PRL expression of the mice that were simultaneously treated with $\mathrm{CAB}$ and miRNA-93 inhibitor was much lower than that of the mice treated with $\mathrm{CAB}$ or
miRNA-93 inhibitor alone $(P<0.05$; Fig. 6D). Western blotting of the MMQ xenograft tumors revealed that the LC3-II protein level was upregulated in the miRNA-93 inhibitor $+\mathrm{CAB}$-treated group $(P<0.01$; Fig. $6 \mathrm{E})$. The TEM assays revealed that the combination of $\mathrm{CAB}$ and miRNA-93 
A

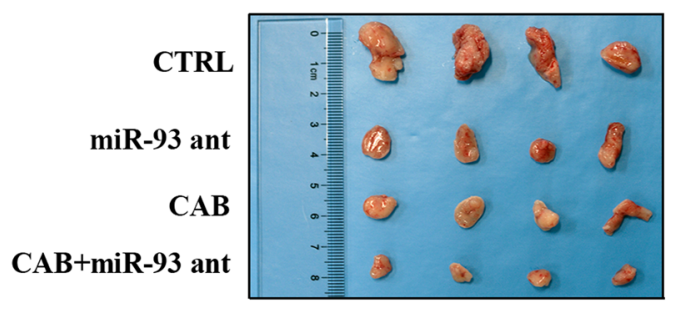

B

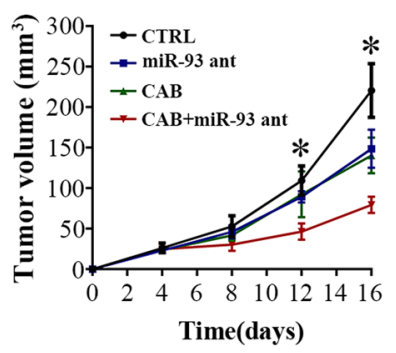

C

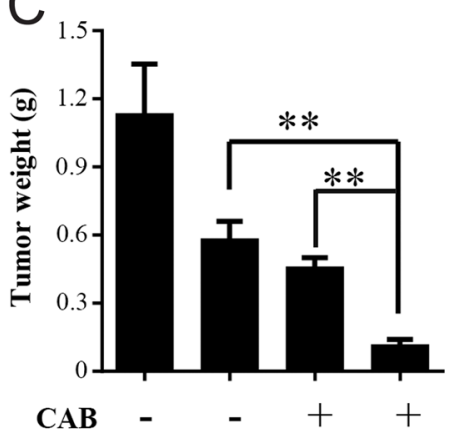

D

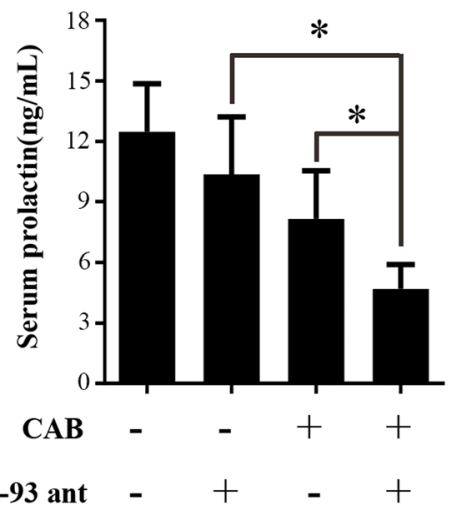

$E$

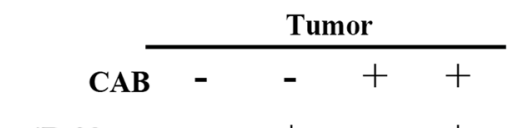

miR-93 ant $\quad-\quad+\quad-\quad+$

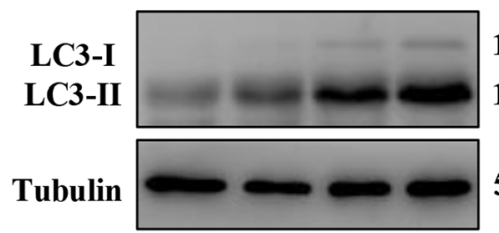

$16 \mathrm{kDa}$

$14 \mathrm{kDa}$

$52 \mathrm{kDa}$

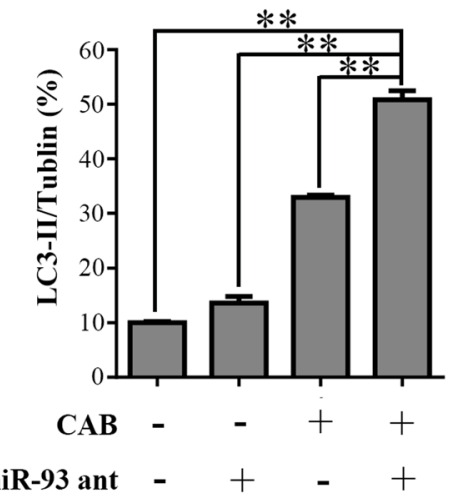

$\mathrm{F}$

Transmission electron microscopy in tumor samples
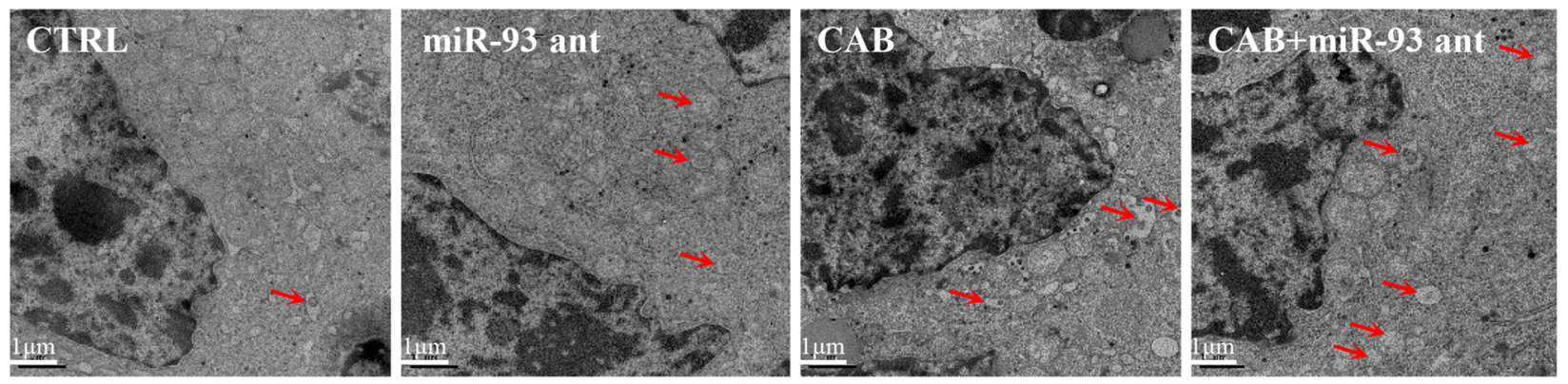

Figure 6

miRNA-93 regulated CAB efficiency in vivo. The representative images for xenograft tumor on the nude mouse (A), the tumor growth curve (B) and tumor weight $(C)(n=4)$. The tumor growth $(B)$ and weight $(C)$ in the CAB + miRNA-93 inhibitor group were significantly inhibited compared with those in the other three groups. (D) The PRL levels in the CAB + miRNA-93 inhibitor group were lower than those in the CAB or miRNA-93 inhibitor alone group. (E) The LC3-II expression was upregulated in the miRNA-93 inhibitor + CAB group compared with that in the other three groups. (F) TEM revealed the formation of autophagosomes (red arrow) in tumor samples obtained from the MMQ xenograft tumors of mice. More autophagosomes were produced in the miRNA-93 inhibitor + CAB group. ${ }^{* *} P<0.01 ; * P<0.05$.

inhibitor produced more autophagosomes in tumor samples obtained from the MMQ xenograft tumors of mice (Fig. 6F), which was consistent with the results of Western blotting. Overall, these results indicated that the miRNA-93 inhibitor enhanced CAB efficiency in vivo by upregulating autophagy.

\section{Discussion}

In the first-line treatment for prolactinomas, $\mathrm{CAB}$ is effective in shrinking the tumor volume, suppressing PRL hypersecretion and restoring gonadal function (Colao et al. 2007). However, some patients are resistant 
to the CAB treatment (Gillam et al. 2006, Wu et al. 2016b), and the treatment of drug-resistant prolactinomas remains a challenging clinical problem.

Accumulating evidence has proven that DAs, such as $\mathrm{CAB}$, play a vital role in inducing autophagic cell death (Wang et al. 2015, Wei et al. 2016). Wang et al. (2015) reported that D2R/D3R agonist(s) might activate autophagy through the BECN1-dependent pathway and inhibit the tumor growth in A549 cells. In addition, D2R antagonists were reportedly involved in autophagy in cardiac myocytes and neuroblastoma cells (Shin et al. 2012, Wei et al. 2016). Likewise, we proved that CAB could induce rat pituitary tumor autophagic cell death (Lin et al. 2015). Thus, the CAB-induced autophagy plays an essential role in inhibiting the development and progression of many tumors.

This study demonstrated for the first time that miRNA-93 directly targeted ATG7 and decreased the autophagic cell death induced by $\mathrm{CAB}$ in rat pituitary tumor cells. In addition, we demonstrated that the miRNA-93 inhibitor could enhance $\mathrm{CAB}$ efficiency in vitro and in vivo. This study elucidated a novel mechanism for the $\mathrm{CAB}$ resistance of prolactinomas and provided a potential therapeutic strategy for CAB-resistant prolactinomas.

Recent studies have demonstrated that miRNA-93 can promote tumorigenesis and drug resistance in multiple cancer types (Li et al. 2017, Wang et al. 2017, Zhang et al. 2017). Wu et al. (2016a) reported that miRNA-93 inhibited Becn1 expression and decreased Becn1-mediated autophagy in leukemia cells. Previously, we showed that miRNA-93 exhibited high expression in DA-resistant prolactinomas (Wu et al. 2014). In addition, one study has shown that the autophagy level is correlated with the sensitivity of cancer cell to chemotherapy (Vazquez-Martin et al. 2009). Our study demonstrated that in pituitary tumor cells, miRNA-93 could decrease $\mathrm{CAB}$ efficiency by downregulating the CAB-induced cell autophagy and leading to drug resistance of tumor cells.

Reportedly, miRNAs can promote the drug sensitivity in glioblastoma by regulating autophagy by targeting ATG7 (Comincini et al. 2013, Zeng et al. 2015). In melanoma cells, the miR-290-295 cluster can inhibit the autophagic cell death by downregulating several essential autophagy genes, including $A T G 7$, which causes drug resistance (Chen et al. 2012). Meanwhile, our previous study established that ATG7 downregulation can reverse the CAB-induced cell death in rat pituitary tumor cells (Lin et al. 2015). ATG7 is a crucial autophagy-related gene that plays a vital role in regulating the chemotherapy efficiency. Consistent with studies mentioned above, our data identified ATG7 as a direct target of miRNA-93 in prolactinomas. Perhaps, ATG7 upregulation can reverse the inhibitory effect of miRNA-93 to CAB-induced cell autophagy and promote the sensitivity of rat pituitary tumor cells to CAB.

This study has several limitations. First, the effect and mechanism of miRNA-93 was studied only in murine cell lines. Hence, the results should be confirmed once human prolactinoma cell lines are established in the future. Second, in addition to the effects on CAB-induced cell autophagy, miRNA-93 might directly influence cell proliferation; thus, the mechanisms warrant further investigation.

\section{Declaration of interest}

The authors declare that there is no conflict of interest that could be perceived as prejudicing the impartiality of the research reported.

\section{Funding}

This work was supported by grants from the Medical Scientific Research foundation of Zhejiang Province, China (WKJ-ZJ-1525); Zhejiang Provincial Natural Science Foundation of China (LY16H160053, LQ17C060002, LY17H160052); Wenzhou Science and Technology Plan Project (Y20170088).

\section{References}

Adams BD, Parsons C, Walker L, Zhang WC \& Slack FJ 2017 Targeting noncoding RNAs in disease. Journal of Clinical Investigation 127 761-771. (https://doi.org/10.1172/JCI84424)

Ahmad N, Haider S, Jagannathan S, Anaissie E \& Driscoll JJ 2014 MicroRNA theragnostics for the clinical management of multiple myeloma. Leukemia 28 732-738. (https://doi.org/10.1038/ leu.2013.262)

Asaduzzaman M, Constantinou S, Min H, Gallon J, Lin ML, Singh P, Raguz S, Ali S, Shousha S, Coombes RC, et al. 2017 Tumour suppressor EP300, a modulator of paclitaxel resistance and stemness, is downregulated in metaplastic breast cancer. Breast Cancer Research and Treatment 163 461-474. (https://doi.org/10.1007/s10549-017-4202-z)

Chen Y, Liersch R \& Detmar M 2012 The miR-290-295 cluster suppresses autophagic cell death of melanoma cells. Scientific Reports 2808. (https://doi.org/10.1038/srep00808)

Cicchini M, Chakrabarti R, Kongara S, Price S, Nahar R, Lozy F, Zhong H, Vazquez A, Kang Y \& Karantza V 2014 Autophagy regulator BECN1 suppresses mammary tumorigenesis driven by WNT1 activation and following parity. Autophagy 10 2036-2052. (https://doi.org/10.4161/ auto.34398)

Colao A, Di Sarno A, Guerra E, Pivonello R, Cappabianca P, Caranci F, Elefante A, Cavallo LM, Briganti F, Cirillo S, et al. 2007 Predictors of remission of hyperprolactinaemia after long-term withdrawal of cabergoline therapy. Clinical Endocrinology 67 426-433. (https://doi. org/10.1111/j.1365-2265.2007.02905.x)

Comincini S, Allavena G, Palumbo S, Morini M, Durando F, Angeletti F, Pirtoli L \& Miracco C 2013 microRNA-17 regulates the expression of ATG7 and modulates the autophagy process, improving the sensitivity to temozolomide and low-dose ionizing radiation 
treatments in human glioblastoma cells. Cancer Biology and Therapy 14 574-586. (https://doi.org/10.4161/cbt.24597)

Duan Z, Gao Y, Shen J, Choy E, Cote G, Harmon D, Bernstein K, LozanoCalderon S, Mankin H \& Hornicek FJ 2017 miR-15b modulates multidrug resistance in human osteosarcoma in vitro and in vivo. Molecular Oncology 11 151-166. (https://doi.org/10.1002/18780261.12015)

Fanini F \& Fabbri M 2016 MicroRNAs and cancer resistance: a new molecular plot. Clinical Pharmacology and Therapeutics 99 485-493. (https://doi.org/10.1002/cpt.353)

Gillam MP, Molitch ME, Lombardi G \& Colao A 2006 Advances in the treatment of prolactinomas. Endocrine Reviews 27 485-534. (https:// doi.org/10.1210/er.2005-9998)

Gozuacik D \& Kimchi A 2007 Autophagy and cell death. Current Topics in Developmental Biology 78 217-245. (https://doi.org/10.1016/S00702153(06)78006-1)

Jiang X, Overholtzer M \& Thompson CB 2015 Autophagy in cellular metabolism and cancer. Journal of Clinical Investigation 125 47-54. (https://doi.org/10.1172/JCI73942)

Leng ZG, Lin SJ, Wu ZR, Guo YH, Cai L, Shang HB, Tang H, Xue YJ, Lou MQ, Zhao W, et al. 2017 Activation of DRD5 (dopamine receptor D5) inhibits tumor growth by autophagic cell death. Autophagy 13 1404-1419. (https://doi:10.1080/15548627.2017.1328347)

Li C, Lyu J \& Meng QH 2017 MiR-93 promotes tumorigenesis and metastasis of non-small cell lung cancer cells by activating the PI3K/ Akt pathway via inhibition of LKB1/PTEN/CDKN1A. Journal of Cancer 8 870-879. (https://doi.org/10.7150/jca.17958)

Lin SJ, Leng ZG, Guo YH, Cai L, Cai Y, Li N, Shang HB, Le WD, Zhao WG \& Wu ZB 2015 Suppression of mTOR pathway and induction of autophagy-dependent cell death by cabergoline. Oncotarget 6 39329-39341. (https://doi.org/10.18632/oncotarget.5744)

Molitch ME 2003 Dopamine resistance of prolactinomas. Pituitary 6 19-27. (https://doi.org/10.1023/A:1026225625897)

Molitch ME 2010 Pituitary gland: can prolactinomas be cured medically? Nature Reviews Endocrinology 6 186-188. (https://doi.org/10.1038/ nrendo.2009.278)

Neesse A \& Gress TM 2015 Emerging role of microRNAs to tackle drug resistance in pancreatic cancer. Gut 64 1842-1843. (https://doi. org/10.1136/gutjnl-2015-309503)

Shin JH, Park SJ, Kim ES, Jo YK, Hong J \& Cho DH 2012 Sertindole, a potent antagonist at dopamine $\mathrm{D}(2)$ receptors, induces autophagy by increasing reactive oxygen species in SH-SY5Y neuroblastoma cells. Biological and Pharmaceutical Bulletin 35 1069-1075. (https://doi. org/10.1248/bpb.b12-00009)

Srivastava AK, Han C, Zhao R, Cui T, Dai Y, Mao C, Zhao W, Zhang X, Yu J \& Wang QE 2015 Enhanced expression of DNA polymerase eta contributes to cisplatin resistance of ovarian cancer stem cells. PNAS 112 4411-4416. (https://doi.org/10.1073/pnas.1421365112)

Tanida I 2011 Autophagy basics. Microbiology and Immunology 55 1-11. (https://doi.org/10.1111/j.1348-0421.2010.00271.x)

Vazquez-Martin A, Oliveras-Ferraros C \& Menendez JA 2009 Autophagy facilitates the development of breast cancer resistance to the antiHER2 monoclonal antibody trastuzumab. PLoS ONE 4. (https://doi. org/10.1371/journal.pone.0006251)

Wang Y, Singh R, Massey AC, Kane SS, Kaushik S, Grant T, Xiang Y, Cuervo AM \& Czaja MJ 2008 Loss of macroautophagy promotes or prevents fibroblast apoptosis depending on the death stimulus.
Journal of Biological Chemistry 283 4766-4777. (https://doi:10.1074/ jbc.M706666200)

Wang JD, Cao YL, Li Q, Yang YP, Jin M, Chen D, Wang F, Wang GH, Qin ZH, Hu LF, et al. 2015 A pivotal role of FOS-mediated BECN1/ Beclin 1 upregulation in dopamine D2 and D3 receptor agonistinduced autophagy activation. Autophagy 11 2057-2073. (https://doi. org/10.1080/15548627.2015.1100930)

Wang SJ, Cao YF, Yang ZQ, Jiang ZY, Cai B, Guo J, Zhang S, Zhang XL \& Gao F 2017 MicroRNA-93-5p increases multidrug resistance in human colorectal carcinoma cells by downregulating cyclin dependent kinase inhibitor 1A gene expression. Oncology Letters 13 722-730. (https:// doi.org/10.3892/ol.2016.5463)

Wei C, Gao J, Li M, Li H, Wang Y, Li H \& Xu C 2016 Dopamine D2 receptors contribute to cardioprotection of ischemic postconditioning via activating autophagy in isolated rat hearts. International Journal of Cardiology 203 837-839. (https://doi. org/10.1016/j.ijcard.2015.11.006)

Wu ZB, Yu CJ, Su ZP, Zhuge QC, Wu JS \& Zheng WM 2006 Bromocriptine treatment of invasive giant prolactinomas involving the cavernous sinus: results of a long-term follow up. Journal of Neurosurgery $\mathbf{1 0 4}$ 54-61. (https://doi.org/10.3171/jns.2006.104.1.54)

Wu ZB, Cai L, Lin SJ, Lu JL, Yao Y \& Zhou LF 2013 The miR-92b functions as a potential oncogene by targeting on Smad3 in glioblastomas. Brain Research 1529 16-25. (https://doi.org/10.1016/j.brainres.2013.07.031)

Wu ZB, Li WQ, Lin SJ, Wang CD, Cai L, Lu JL, Chen YX, Su ZP, Shang HB, Yang WL, et al. 2014 MicroRNA expression profile of bromocriptineresistant prolactinomas. Molecular and Cellular Endocrinology 395 10-18. (https://doi.org/10.1016/j.mce.2014.07.014)

Wu X, Feng X, Zhao X, Ma F, Liu N, Guo H, Li C, Du H \& Zhang B $2016 a$ Role of Beclin-1-Mediated autophagy in the survival of pediatric leukemia cells. Cellular Physiology and Biochemistry 39 1827-1836. (https://doi.org/10.1159/000447882)

Wu ZR, Zhang Y, Cai L, Lin SJ, Su ZP, Wei YX, Shang HB, Yang WL, Zhao WG \& Wu ZB $2016 b$ Long-term clinical outcomes of invasive giant prolactinomas after a mean ten-year followup. International Journal of Endocrinology 2016 8580750. (https://doi. org $/ 10.1155 / 2016 / 8580750)$

Xiong J 2015 Atg7 in development and disease: panacea or Pandora's Box? Protein and Cell 6 722-734. (https://doi.org/10.1007/ s13238-015-0195-8)

Zeng Y, Huo G, Mo Y, Wang W \& Chen H 2015 MIR137 regulates starvation-induced autophagy by targeting ATG7. Journal of Molecular Neuroscience 56 815-821. (https://doi.org/10.1007/s12031015-0514-9)

Zeng HC, Bae Y, Dawson BC, Chen Y, Bertin T, Munivez E, Campeau PM, Tao J, Chen R \& Lee BH 2017 MicroRNA miR-23a cluster promotes osteocyte differentiation by regulating TGF-beta signalling in osteoblasts. Nature Communications 8 15000. (https://doi.org/10.1038/ ncomms15000)

Zhang W, Xu J, Shi Y, Sun Q, Zhang Q \& Guan X 2015 The novel role of miRNAs for tamoxifen resistance in human breast cancer. Cellular and Molecular Life Sciences 72 2575-2584. (https://doi.org/10.1007/s00018015-1887-1)

Zhang R, Guo Y, Ma Z, Ma G, Xue Q, Li F \& Liu L 2017 Long non-coding RNA PTENP1 functions as a ceRNA to modulate PTEN level by decoying miR-106b and miR-93 in gastric cancer. Oncotarget 8 26079-26089. (https://doi.org/10.18632/oncotarget.15317)

Received in final form 4 September 2018

Accepted 24 September 2018

Accepted Preprint published online 24 September 2018 https://joe.bioscientifica.com https://doi.org/10.1530/JOE-18-0203 (c) 2019 Society for Endocrinology Published by Bioscientifica Ltd.
Printed in Great Britain 